\title{
Analytical characterization of viruses
}

\author{
Joseph Zaia ${ }^{1}$ \\ Received: 13 September 2021 / Accepted: 13 September 2021 / Published online: 1 October 2021 \\ (C) Springer-Verlag GmbH Germany, part of Springer Nature 2021
}

The World Health Organization declared the SARSCoV-2 pandemic on Mar 11, 2020. Prior to this time, much of the concern regarding pandemic viruses was focused on the influenza A virus. At the beginning of the pandemic, it was known that coronaviruses have circulated within the human population for at least several thousand years. The trajectory of SARS-CoV-2 was not known, and the virus was believed to undergo genetic mutation at a much lower rate than the influenza A virus. Notwithstanding these early assessments, we now know that SARS-CoV-2 can mutate to become more infectious and evade immunity, with serious consequences for the efficacy of vaccines and therapeutic treatments.

These concerns highlight the importance of characterizing the structures of viruses and how they evolve, adapt to infect humans, and maintain the ability to circulate in the human population. We already appreciate that viruses undergo genetic mutations that cause amino acid substitutions that influence virus antigenicity, immunogenicity, and fitness. But we cannot predict the immunological properties of a virus or its ability to infect host cells from its genomic sequence. The ability to do so would enhance surveillance of emerging viruses.

Viral genomes are small and they cannot replicate without host cells. In a sense, viruses can be considered molecular machines consisting of nucleic acids, proteins, lipids, and carbohydrates. We seek to understand how viruses recognize their

Published in the topical collection Analytical Characterization of Viruses with guest editor Joseph Zaia.

Joseph Zaia

Jzaia@bu.edu

1 Department of Biochemistry, Boston University Medical Campus, 670 Albany St., Rm. 509, Boston, MA 02118-2646, USA hosts, enter cells, hijack the biosynthetic machinery, and replicate. These properties depend on the interactions among virus particles, host receptors and co-receptors, and cell membranes that depend on the overall structures of the viral proteins and glycoproteins. The adhesive properties of viral spike proteins depend on glycosylation, the glycosylation sites for which evolve as the virus circulates in the human population. In addition, each glycosylation site is heterogeneous, reflecting a distribution of related glycan structures that give rise to a distribution of adhesive properties. At the present time, we can characterize the glycosylated forms of different virus preparations. Yet we do not know the structures of the virions that infect human airway cells.

This topical collection highlights emerging analytical methods for characterizing viruses. These methods include proteomics to assess viral heterogeneity. Glycomics is used to characterize the glycans released from viral spike proteins. Glycoproteomics is used to assign the site-specific glycosylation necessary to understand the physicochemical and immunological properties of spike proteins. The characterization of viral proteins using top-down methods helps define the glycoproteoform distribution present in a virus sample. Remarkably, whole virus particle characterization helps define the structure of the complete macromolecular assembly.

I thank the authors for their contributions to this topical collection and the referees for their timely reviews of the submitted manuscripts. I thank the editorial office for their excellent guidance and support.

Publisher's note Springer Nature remains neutral with regard to jurisdictional claims in published maps and institutional affiliations. 


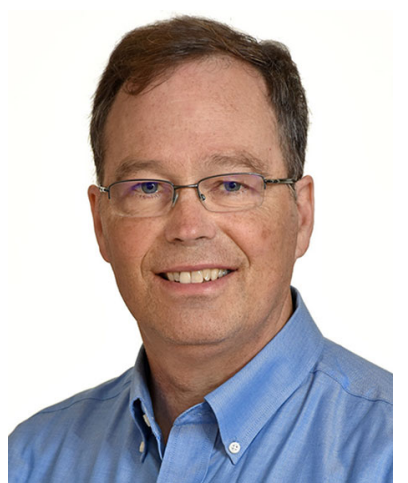

Joseph Zaia 's research group focusses on the analytical biochemistry of glycoproteins and proteoglycans. They have developed methods for combined glycomics, proteomics, and glycoproteomics from tissue slides. They have produced a set of bioinformatics tools for the interpretation of mass spectrometry-based glycomics and glycoproteomics data sets. Their major goals are to track changes in protein glycosylation during biological mechanisms. Specific projects include (i) mapping changes in extracellular matrix protein glycosylation during brain disease mechanisms and (ii) analysis of the roles of viral spike protein glycosylation in the evolution of pathogenic enveloped viruses. Dr. Zaia has published more than 150 articles in peer-reviewed scientific journals and has an $H$-index of 51. He is an editor of Analytical and Bioanalytical Chemistry. 